\title{
LOS “GIROS” DE LA UNIVERSIDAD Y EL DESARROLLO LOCAL
}

\author{
Jorge R. Núñez Jover (Universidad de La Habana)* \\ jorgenjover@rect.uh.cu \\ Aurora Fernández González (Universidad de La Habana)** \\ aurora@reduniv.edu.cu \\ José Lázaro Hernández Gil (Universidad de La Habana)*** \\ jlazaro@rect.uh.cu
}

Recibido: 24/07/2012 Aceptado: 20/10/2012

\section{Resumen}

En la década que corre se afirma de modo más apremiante que nunca la necesidad de articular las universidades al tejido productivo y social. Cuba, con sus problemáticas específicas, también enfrenta este desafío. La mayor novedad quizás está en el hecho de que se trata ahora de la articulación de los esfuerzos universitarios en contextos locales ¿Estamos preparados para ello? ¿Qué hace la educación superior para avanzar en ese terreno? Comenzaremos por hacer un comentario general sobre el papel de las universidades en ciencia, tecnología e innovación; luego nos detenemos en la evolución de la universidad cubana y finalmente presentaremos un proyecto en el que venimos trabajando.

\section{Palabras clave}

Universidad - Educación superior - Conocimiento - Desarrollo social - Desarrollo local.

* Doctor en Filosofía. Licenciado en Química (Universidad de La Habana). Director de Posgrado de la Universidad de La Habana. Cordinador de la Cátedra Ciencia, Tecnología y Sociedad de la Universidad de La Habana.

** Doctora en Ciencias Técnicas. Máster en Investigación de Operaciones. Ingeniera Industrial. Universidad de La Habana. Profesora Títular y profesora de Merito del Instituto Superior Politécnico José Antonio Echevarría (ISPJAE) de La Habana. Profesora de la Cátedra Ciencia, Tecnología, Sociedad e Innovación de la Universidad de La Habana.

${ }^{* * *}$ Máster en Informática. Graduado de la Profesoral Superior de Historia y la Licenciatura en Filosofía. Metodólogo Asesor de la Universidad de La Habana. 


\begin{abstract}
The need to connect universities to the productive and social web is more urgent than ever. Cuba, with its specific characteristics, also faces this challenge. The biggest novelty is maybe the fact that we face now the articulation of university to local development. Are we prepared for this? What is higher education doing to advance in this field? In the paper we start by making a general comment about the role of the universities in science, technology and innovation, analyzing in detail the evolution of Cuban university and finally we will present a project in which we are working.
\end{abstract}

\title{
Key words
}

University - Higher education - Knowledge - Social development - Local development.

\section{Introducción}

En el mundo tiene un gran peso la idea de que el conocimiento es determinante para crear riqueza, mejorar la salud, cuidar el medio ambiente y lidiar con problemas sociales de todo tipo como la pobreza, la exclusión social, la violencia, entre otros.

A las universidades corresponde un papel relevante en la generación de ciencia, tecnología, innovación (CTI) y formación de alto nivel (1). La bibliografía sobre el tema refleja ese consenso (Sousa Santos, B (2008); Lynn Meek, V., U. Teichler, M-L. Kearney (2009); Santelices, B, 2010; Albornoz, M y J.A López Cerezo, 2010, Núñez, J, 2010). Ello explica que el tema fuera objeto de atención en la Conferencia Regional de Educación Superior para América Latina y el Caribe (Cartagena de Indias, Colombia, 2008) [2].

Sin embargo, si se examinan las políticas universitarias orientadas a promover actividades de CTI y formación de alto nivel es posible apreciar diversos enfoques que incorporan diferentes presupuestos epistemológicos y axiológicos.

Esquematizando un poco, es posible observar políticas que se encuadran dentro de enfoques académicos más tradicionales, orientados a promover la investigación científica conducida por los objetivos de verdad y objetividad, rigor, racionalidad, cuyo destino primordial es el consumo por los pares académicos y su expresión son las publicaciones, sin demasiado interés por otros usos económicos y sociales.

Otras políticas se interesan por la capitalización del conocimiento, privilegian el vínculo universidad-empresa y en correspondencia con el Modelo de la Triple Hélice (Etzkowitz, H y L. Leydesdorff, 1997) defienden el desempeño empresarial de la universidad y los mismos investigadores (3).

En las últimas décadas las universidades han procurado articular cada vez más directamente sus capacidades de formación e investigación con la economía y la sociedad. Para ello han modificado sus agendas de formación 
e investigación y creado estructuras nuevas como parques tecnológicos, incubadoras de empresas, oficinas de transferencia de tecnología. En suma, han gestado nuevos arreglos institucionales y legales; han creado nuevos incentivos y se han diversificado las culturas (académica, económica, burocrática) que actúan al interior del mundo universitario.

Se viene hablando con fuerza de una "tercera misión" empresarial de la universidad que se suma a las funciones de formación e investigación. En su formulación hay mucha ambigüedad. Se suelen incluir dimensiones tales como: 1) La transferencia de conocimiento incorporado en estudiantes de doctorado y graduados (transferencia de competencias entrenadas a través de la investigación a la industria y los servicios públicos orientados por misiones). 2) La propiedad intelectual, entendida como conocimiento codificado producido por la universidad y su gestión (patentes, copyright). 3) Spin-offs: transferencia de conocimiento a través del emprendedurismo. 4) Contratos con la industria a través de la coproducción de conocimiento y su circulación a la industria. Se supone que es el principal atractivo de las universidades para los actores económicos. 5) Contratos con cuerpos públicos, para satisfacer la dimensión de servicio público de las actividades de investigación. 6) La participación en la elaboración y/o implementación de políticas, entre otros. La tendencia a acercar la universidad a los sectores productivos parece irreversible en un contexto donde el conocimiento incrementa su importancia económica y social. Es obvio, sin embargo, que la "tercera misión" tiene una orientación netamente económica y tiende a centrarse en las llamadas "ciencias duras" y la tecnología.

Existen también políticas institucionales que sin descartar necesariamente a las anteriores se interesan por la orientación social de sus prácticas. Les interesa fomentar la interacción universidad-sociedad en un sentido amplio, se guían por objetivos de pertinencia social y despliegan agendas centradas en necesidades sociales.

Ese tipo de políticas se interesan especialmente por el papel del conocimiento, la ciencia, la tecnología y la innovación en los procesos de inclusión social y cohesión social (Sutz, 2010; OEI, 2012). La inclusión social como objetivo de las actividades de CTI ha sido incorporada recientemente en la agenda de discusión. Esto se relaciona con el hecho de que el avance del conocimiento y de sus aplicaciones no parece atenuar la desigualdad y la pobreza. Al incorporar la solución de problemas sociales y el tema de la inclusión social a las agendas de CTI de las universidades-y más allá de ellas- se crean oportunidades a la producción, distribución y uso del conocimiento orientados a demandas frecuentemente insatisfechas.

Grupos de investigadores asociados a la red UNIDEV (4) hemos valorado el concepto de "universidades desarrolladoras" que quiere subrayar la articulación de las universidades al desarrollo social, no solo a través del mercado, la competitividad y la ganancia, en suma, haciendo negocios, sino implicándose como 
actor relevante del desarrollo económico y social en todas sus dimensiones. Se subraya que el conocimiento universitario es también un bien cultural que nos debe ayudar a generar inclusión social, promover salud, resolver problemas sociales, cuidar el medio ambiente, entre otros muchos otros beneficios.

La trayectoria de la educación superior cubana puede ser vista como un esfuerzo por construir capacidades en CTI y alinearlas con las necesidades del desarrollo social. A continuación sintetizaremos sus principales transformaciones y nos detendremos en el esfuerzo que hoy desarrollamos por conectar educación superior y desarrollo local, en particular el modelo conceptual que hemos construido.

\section{Transformaciones en la educación superior cubana: nuevas políticas, nuevas agendas}

Mencionemos rápidamente algunos de los rasgos que caracterizan la educación superior cubana: cobertura nacional a través de una red de instituciones que abarcan todo el territorio nacional y ofrecen programas de formación de nivel adecuado y de acceso gratuito, siempre financiados por el Estado; una parte no desestimable de los profesores y estudiantes están involucrados en actividades de ciencia, tecnología e innovación; la formación de grado se continúa mediante un sistema de formación posgraduada que asume la pertinencia social como valor integrante de la calidad; muchas universidades han ido creando mecanismos para facilitar la innovación tecno productiva y social mediante la articulación estrecha con el sistema productivo y las comunidades.

Desde tal sistema universitario se intenta atender grandes demandas de nuestra sociedad, incluida la necesidad apremiante de movilizar todos los potenciales productivos del país y la urgencia de utilizar con ese fin el potencial humano, de conocimientos e innovación, que el país tiene. Dentro de la decisión de descentralizar decisiones y recursos que acompaña al modelo económico y social en gestación la dimensión territorial / local adquiere mayor relevancia que en cualquier período anterior.

La pregunta es si nuestras universidades están preparadas para actuar como "universidades desarrolladoras" en semejante contexto de demandas renovadas. Para responder a esta pregunta es necesario repasar el camino recorrido por la educación superior cubana.

De modo esquemático es posible asumir que la universidad ha vivido en las cinco décadas que nos separan de la Reforma de la Enseñanza Superior de 1962 un conjunto de cambios relevantes que la ayudan a afrontar las nuevas demandas. Llamaremos "giros" a esos cambios y los resumimos en cinco.

El primero de ellos fue el "giro a la investigación" asociado a la Reforma de 1962 con lo cual la investigación pasó a convertirse paulatinamente en componente de la vida universitaria (5). El clímax en esa transición fue la creación del Centro nacional de investigaciones científicas (CENIC), el día primero de julio 
de 1965. El CENIC pasó a formar parte de la Universidad de La Habana (UH) en 1966, convertido en institución clave en la formación de investigadores. El modelo de investigación que intentó construir la universidad cubana desde los tiempos de la Reforma Universitaria de 1962, puede denominarse como un modelo "interactivo", atento a las necesidades sociales.

A la etapa que media entre 1966 y 1974 la denominamos como el "giro a políticas orientadas a la investigación aplicada". En la medida que las capacidades científicas y la organización institucional fueron madurando, fue consolidándose una política universitaria-influida y respaldada por los más altos niveles de Gobierno-orientada a una articulación cada vez más vigorosa con los programas económicos y sociales del país. Ejemplos de aquella fructífera etapa fueron el Programa Azúcar que incorporaba problemas de agricultura, cosecha, transportación y procesamiento industrial de la caña. Incluía más de 100 proyectos con intervención de las Ramas Agrícola, Agroindustrial, Química, Mecánica y Computación. También constituye un buen ejemplo la fabricación de la primera computadora cubana, con la denominación CID-201-A. En aquellos años se construyó activamente una política científica universitaria que permitió el crecimiento notable de la ciencia universitaria y consolidó sus nexos con la sociedad y el sector productivo. El país defendía un proyecto de desarrollo que reclamaba a la ciencia universitaria.

Los cambios institucionales y las contingencias económicas que tuvieron lugar a inicios de los 70s y la entrada de Cuba al Consejo de Ayuda Mutua Económica (CAME) [6], limitaron el ritmo de crecimiento de la ciencia universitaria, aunque se incrementó el proceso de formación de estudiantes de grado y posgrado, lo que permitió continuar acumulando capacidades.

Hacia la primera mitad de los 80 s, la situación económica de la URSS y las posibilidades del modelo económico del CAME condujeron al gobierno cubano a la búsqueda de alternativas. Las capacidades que el país había creado en ciencia y la tecnología, se apreciaron como una oportunidad. Ello explica que desde la mitad de los 80 s y sobre todo a inicio de los 90 s comenzó lo que denominamos el "giro a la innovación" asociado a la idea de fomentar organizaciones que "cerraran el ciclo" generando innovaciones de importancia social y económica. Se realizaron importantes inversiones en las universidades y se crearon instituciones volcadas a la innovación. La primera de todas fue el Instituto de Materiales y Reactivos (IMRE) de la Universidad de La Habana creado en 1985, estrechamente vinculado a la industria electrónica y luego surgieron otro centros en la propia Universidad de La Habana (UH), Universidad Central de Las Villas (UCLV), Universidad de Oriente (UO), Universidad de Matanzas "Camilo Cienfuegos" (UMCC), entre otras. Todos han venido acumulando experiencias en materia de innovación, con resultados de variada importancia (Núñez, J; F. Castro, 2005). 
El declive y posterior desaparición del socialismo europeo, que proporcionaba a Cuba las fuentes principales de formación posgraduada, condujo en los 90s a cambios en las políticas de educación superior que llevaron a lo que denominamos el "giro al posgrado" que permitió consolidar el sistema nacional de posgrado. En lo adelante la formación de posgrado se hizo más dependiente de las capacidades nacionales, a la vez que modificó radicalmente su inserción internacional.

Recapitulemos lo visto hasta aquí: en las décadas transcurridas el país amplió su sistema universitario, bien respaldado por el sistema educativo precedente; formó profesionales de buen nivel; creó una base científica nacional; desarrolló la investigación universitaria, una parte de la cual se volcó hacia esfuerzos de innovación y consolidó un sistema nacional de posgrado. De una u otra forma, el liderazgo político procuró siempre alentar la educación, el conocimiento, la ciencia.

Todo ello realizó en condiciones económicas casi siempre complejas, en un contexto permanentemente influido por el bloqueo económico de los Estados Unidos. Con la caída de la URSS, al inicio de la década de los 90 s, la situación económica se volvió en extremo crítica y con ella se acumularon no pocos problemas sociales. Uno de ellos era el descenso notable de las matrículas universitarias. A inicios de la pasada década el gobierno cubano decidió ampliar el acceso a las universidades a través de la extensión de la red de instituciones de educación superior del país, creando espacios de educación superior en los municipios. Se trataba de un nuevo esfuerzo por extender los beneficios de la educación a la población, cuyo referente más remoto es la Campaña de Alfabetización de 1961, seguida de la Reforma Universitaria de 1962.

Así, en la década más reciente hemos asistido al "giro territorial". Comenzó con la municipalización de la educación superior orientada a garantizar el acceso pleno a la educación superior en un intento por saldar una deuda social acumulada. (Núñez, J., F. Benítez., D. Hernández; A. Fernández, 2008).

A partir de 2006 grupos de profesores universitarios nos agrupamos en el Programa de "Gestión universitaria del conocimiento y la innovación para el desarrollo" (GUCID) [7] que nació bajo la idea de que la contribución de la educación superior al desarrollo local y territorial no se agotaba con la fórmula del acceso a los estudios. Se comprendió rápidamente que podía ser un instrumento para promover desarrollo local y que las universidades podían jugar un rol fundamental en ese proceso (Núñez, J., L.F Montalvo; I, Pérez, 2007).

En su funcionamiento GUCID actúa como una red social de conocimientos que enlaza los diversos actores de la educación superior cubana (universidades, centros de investigación, redes, programas, centros universitarios municipales y filiales universitarias municipales) con el propósito de fomentar el desarrollo local a través del esfuerzo de las instituciones y organizaciones universitarias. 
Hasta fecha reciente el tema del desarrollo local y territorial ocupaba en Cuba un lugar secundario. Sin embargo, los Lineamientos de la Política Económica y Social aprobados en 2011 que definen las principales políticas del país, enfatizan la importancia del desarrollo territorial, local.

Construir desarrollo local es una empresa mayúscula que requiere de muchos conocimientos y tecnologías y de marcos institucionales y legales que apenas se están creando. En principio el desarrollo local supone:

- Proveer oportunidades: acceso a opciones en el mercado laboral, a créditos, propiedades, infraestructura adecuada, educación, salud, entre otros.

- Garantizar seguridad: cobertura total de las necesidades básicas para una vida digna; reducción de desigualdades, riesgos y vulnerabilidades.

- Empoderamiento de los actores locales: descentralización de funciones, autoridad y recursos e implementación de mecanismos participativos para la definición de la agenda social y de desarrollo del territorio.

- Sustentabilidad de las iniciativas locales: creación de la capacidad institucional y de conocimientos.

- Fortalecimiento y rescate de la economía local: multiplicación y diversificación de agentes de poder económicos locales, sus interrelaciones y que mantengan un compromiso comunitario.

- Capacidad de los actores locales para realizar una gestión estratégica: formulación, implementación, evaluación, de políticas, programas, proyectos.

- Capacidad de las sociedades locales para identificar posibilidades endógenas de desarrollo y atracción de recursos exógenos.

Ese desarrollo requiere de conocimientos, tecnologías e innovación. No es imaginable el desarrollo económico y social de un municipio o del país en su conjunto, al margen del aprendizaje, la tecnología y la capacidad de innovación.

La pregunta es qué instituciones tienen esas capacidades para apoyar el desarrollo municipal.

\section{Los actores}

Como mencionamos antes, en la década anterior se crearon en todos los municipios del país espacios de educación superior, las llamadas Sedes Universitarias Municipales (SUM), ahora denominadas Centros Universitarios Municipales (CUM). El surgimiento de tales organizaciones representó una verdadera innovación que permitió crear en todos los municipios instituciones de conocimiento que reúnen una parte importante de los profesionales de los territorios y que tienen capacidad de impulsar las estrategias de desarrollo municipales a través del trabajo en red con universidades, centros de investigación, redes de conocimiento y programas. Tales vínculos facilitan el flujo de conocimientos y tecnologías a los municipios. 
GUCID asume entonces que los Centros Universitarios Municipales son actores colectivos capaces de impulsar la gestión del conocimiento volcada al desarrollo local. Pero las potencialidades de la educación superior no se reducen a esos actores. Tenemos también las universidades que realizan tareas de formación, de investigación y de extensión y cuentan con grupos capaces de transferir tecnologías. Finalmente contamos con las redes (dedicadas a temas como energías alternativas, materiales de construcción con ecomateriales, agoecología y muchas más) que actúan en numerosos municipios del país. Esos actores, trabajando en red y enlazados a los gobiernos, las empresas y demás actores de importancia para el desarrollo local pueden impulsar la innovación y el desarrollo.

El país tiene necesidades apremiantes de movilizar todos los potenciales productivos del país. La tarea de GUCID es promover las capacidades de gestión y sinergias entre los actores mencionados y conectarlos vigorosamente con las necesidades del desarrollo local.

El trabajo de GUCID se apoya en tres ideas:

1. La educación superior es clave en la producción, distribución y uso del conocimiento orientado al desarrollo.

2. Gestionar conocimiento es conectar los actores que disponen de conocimientos y tecnologías con aquellos que tienen necesidades de los mismos para satisfacer necesidades sociales. De la experiencia pionera del municipio de Yagüajay, Agustín Lage (Lage, 2006) extrajo una fórmula que nos sirve de orientación: Según él, la gestión del conocimiento supone: a) identificación de los actores; b) construcción de redes; c) construir conectividad; d) estimular y organizar interacciones; e) crear en las empresas capacidad de asimilar conocimientos, tecnologías; f) armar "ciclos cerrados" a través de la dirección por proyectos; g) implementar la capacitación para toda la vida; h) seleccionar, capacitar y evaluar los cuadros; i) Construir infraestructura (informatización entre otras exigencias); j) Construir y evaluar indicadores.

3. Innovar es dar solución creativa-basada en el conocimiento-a problemas (económicos, sociales) en ambientes productivos. Esas soluciones deben generar inclusión, cohesión e integración social.

\section{El modelo contexto-céntrico y los agentes locales del conocimiento}

El llamado "modelo ofertista" basado en el "modelo lineal de innovación", según el cual las instituciones productoras de conocimiento, las universidades entre ellas, generan conocimiento a través de la investigación, el cual en su momento generará tecnologías e innovaciones, es un modelo superado en la teoría y en la práctica. 
Una teoría que anuncia la superación del modelo ofertista es la teoría del "modo 2" de producción de conocimientos, modelo que se opone al "modo 1" propio del mundo académico tradicional (Gibbons et al, 1997). El llamado "modo 2", es resultado del estudio de los cambios operados en la relación CienciaSociedad en Estados Unidos, Europa, Japón, entre otros. "Modo 2" toma nota de algunas mutaciones fundamentales: el conocimiento pasa a ser producido en el "contexto de su aplicación", es decir, a diferencia de lo que sugerían los modelos ofertistas tradicionales ahora el conocimiento, en su mayoría, se produce orientado a su aplicación. La innovación aparece como clave. El nuevo modelo rompe también con la idea del monopolio cognitivo por parte de unas u otras instituciones, por ejemplo las universidades, y asume que el conocimiento esta socialmente distribuido, factor que influye en la necesidad de trabajar en redes, no solo académicas. Desde este modelo es posible también apreciar la importancia del compromiso compartido, el papel de los valores y las intencionalidades sociales en la producción del conocimiento. La interdisciplinariedad aparece como el estilo de trabajo científico apropiado para trabajar en el contexto de aplicación. Como resultado de todo ello, los criterios de calidad del trabajo que tradicionalmente hemos llamado científico, también serán cambiados.

En ese tipo de modelo, el contexto aparece como relevante en la definición de prioridades, en la construcción de redes. A partir de ese modelo, Souza (Souza, Cheaz y Calderón, 2001) ha introducido el concepto de "modelo contextocéntrico" (8). Con esta denominación se quiere subrayar que el contexto debe ser la clave en la construcción y orientación social del conocimiento. Pero se quiere decir algo más. La teoría del "modo 2" como otras, tienen un fuerte acento económico, con énfasis claramente empresarial. Sin embargo, el contexto no son sólo las empresas y lo que se puede esperar del conocimiento, no son exclusivamente beneficios económicos. Esos beneficios, además deben alcanzar a las grandes mayorías. Por todo ello, la idea de modelo contexto-céntrico es afín al argumento que aquí presentamos.

Este enfoque nutre el modelo de investigación por el cual ha trabajado la educación superior cubana, atento a las necesidades sociales. La municipalización de la educación superior y el interés por el nexo educación superiordesarrollo local conducen a que ese modelo interactivo se exprese en los territorios a través del modelo contexto-céntrico.

\section{El conocimiento necesario para el desarrollo}

La dimensión local es muy relevante para las estrategias de desarrollo social y tiene una muy alta significación para los procesos de construcción del conocimiento. A lo primero parece oponerse una visión excesivamente centralista que subestima las estrategias locales, con sus singularidades, privilegiando políticas verticales y homogéneas. A la idea de la relevancia del contexto en 
la definición de las prácticas epistémicas parece oponerse la más tradicional idea de la universalidad de la ciencia.

En realidad la idea de la relevancia del contexto, apunta a una epistemología diferente: subraya que la producción, distribución y uso de los conocimientos, tecnologías, tiene siempre lugar en contextos particulares, con sus singularidades económicas, culturales, valorativas, y que esos contextos deben modelar sus prioridades y desarrollo.

Los CUM pueden realizar una sensible contribución a incentivar la proyección local del conocimiento y la innovación, ampliando su capacidad de fomentar el bienestar humano en los territorios. Allí, al nivel del territorio, a través del aprovechamiento de los recursos propios y externos, debe producirse un encuentro mucho más íntimo entre estrategias de conocimiento, prioridades del desarrollo y bienestar humano resultante. La calidad de vida de las personas supone atender el crecimiento y el desarrollo económico, producción de alimentos, cobertura y calidad de la educación, salud, empleo, vivienda, cultura, sostenibilidad ambiental, acceso y calidad del agua, viales y transporte, servicios a la población, aspectos legales y todo lo relativo a la participación social en las tareas de gobierno (personas como agentes, no pacientes).

Obsérvese que hablamos de conocimiento y no de ciencia, investigacióndesarrollo u otros términos. Los CUM pueden hacer investigación con alguna pretensión de originalidad que puede servir para identificar y buscar respuestas a problemas regionales. El cuerpo docente de los CUM y los alumnos pueden aportar mucho a ese propósito. Hay muchos problemas cotidianos cuya solución se puede beneficiar de las investigaciones, cuanto más orientadas a fines prácticos, mejor.

Pero las acciones a realizar no se refieren exclusivamente a esto. La gestión del conocimiento consiste en gran medida en la identificación de problemas locales que requieran del conocimiento para su solución así como la identificación de las organizaciones o personas que pueden aportarlo para luego construir los nexos, las redes y los flujos de conocimiento que permitan la asimilación, evaluación, procesamiento y uso de esos conocimientos. Los CUM deben actuar como agentes relevantes en la construcción social del conocimiento y el establecimiento de las conexiones que permitan los flujos y el empleo del conocimiento.

Pareciera una tarea menor pero está lejos de serlo. La gestión de conocimiento en el nivel local plantea retos de gran interés. El primero es de naturaleza epistemológica. Con frecuencia los problemas a abordar son de carácter complejo y reclaman un abordaje multi o interdisciplinario. Problemas de alimentación, vivienda, salud, violencia familiar u otros en el nivel local, requieren la integración de varias disciplinas en la búsqueda de respuestas. En muchos casos las soluciones están en una combinación inteligente de los conocimientos existentes. 
El segundo reto consiste en que el conocimiento que se requiere es un conocimiento integrado a la práctica; su búsqueda se genera para solucionar un problema práctico y va al encuentro de él. Buena parte del conocimiento necesario para resolver ese problema, existe y se trata más bien de transferirlo; al hacerlo, la singularidad de las circunstancias locales exigirá buenas dosis de creatividad. Sin embargo, parece claro que el aprendizaje por parte de los actores locales aparece en primer plano dentro de la actividad cognoscitiva que la práctica local reclama. Una pieza clave de esa estrategia lo constituye el programa de educación continua de los profesionales del territorio. Y aquí tenemos un tercer desafío a destacar: el nexo entre innovación y aprendizaje. Esta consideración parece acrecentar el papel de los CUM en la estrategia de gestión del conocimiento y la innovación de los territorios.

La complejidad epistemológica asociada a lo que hemos llamado modo de producción "contexto-céntrico" de conocimiento la ha resumido Agustín Lage (Lage, 2006) a través de la caracterización de lo que él llama "conocimiento relevante", el cual tendría los siguientes rasgos: a) Colectivo (incorporado a las organizaciones); b) Combinatorio (fuentes y disciplinas diversas); c) Concreto (vinculado a las aplicaciones); d) Local; e) Tácito (frecuentemente no estructurado). En efecto, esta síntesis ilumina bastante bien el tipo de conocimiento que deberán gestionar los CUM para atender los problemas del territorio.

También ayuda a comprender la problemática epistemológica la tipología de Lundvall (Lundvall, 2000). Los conocimientos útiles para el desarrollo han sido resumidos por él a través de unas pocas palabras: know what, (alude al manejo de información), know how (saber cómo, más bien de carácter tecnológico, a veces tácito), know why (se refiere al conocimiento científico, codificado, frecuentemente sistemático y el know who. Este último es decisivo para la función que los CUM deben desarrollar. Se trata del conocimiento que los individuos y las instituciones acumulan acerca de las personas que poseen conocimientos que pueden ser útiles en un momento dado, para la solución de un determinado problema. No solo es importante tener conocimientos científicos, técnicos para resolver con la propia capacidad un problema dado, es preciso "saber quién sabe" y acudir a esa fuente. Esa capacidad es esencial para el desarrollo.

A la idea de Lundvall, sobre los tipos de conocimiento útiles para el desarrollo, habría que incorporarle un aspecto: no solo es importante el "saber cómo" que denominamos tecnológico, asociándolo a alguna base científica; convendría también aceptar el valor del conocimiento cotidiano, de la etnociencia, apoyado en tradiciones culturales que con frecuencia no son reconocidos en el campo científico, aunque no tienen por que ser considerados anticientíficos. Los CUM pueden jugar un papel importante en la recuperación de esos saberes que son parte de la cultura.

Para GUCID los CUM son muy importantes en esta estrategia de la educación superior volcada al desarrollo local, aunque como vimos, no actúan en 
solitario sino junto a otros actores de la educación superior y externos a ella. Sin embargo, en las experiencias venimos acumulando, los CUM, unos en mayor medida que otros, cumplen funciones relevantes. Estas son:

1. Ayudar a pensar lo local y a valorizar el papel que el conocimiento juega en su desarrollo. Fomentar el imaginario innovativo.

2. Interactuar permanentemente con el gobierno.

3. Participar en construcción de planeación de estrategias de desarrollo apoyadas en el conocimiento.

4. Favorecer la articulación de las formaciones al desarrollo local: formación en los niveles de grado y posgrado, atracción de estudiantes del municipio que se forman en sedes de los centros de la educación superior (CES), formación de técnicos, oficios.

5. Apoyar la Gestión de proyectos (capacitación, elaboración, evaluación, etc.)

6. Participar en la construcción de estrategias de formación del potencial humano.

7. Participar en la formación diferenciada de cuadros, reservas y otros actores.

8. Construir conexiones entre actores del municipio (o que operan en el municipio) para producir, distribuir y usar el conocimiento.

9. Construir conexiones del territorio con entidades de ciencia e innovación tecnológicas (ECIT), centros de educación superior (CES); permitiendo la construcción de redes que favorezcan flujos de conocimientos y tecnologías para resolver problemas del desarrollo local.

10. Valorizar, conservar, difundir el conocimiento tradicional.

11. Servir de apoyo a las redes agropecuarias, de construcción de viviendas, energía y otras que despliega la educación superior por todo el país y las que operan otros organismos.

12. Elaborar y utilizar indicadores que permitan evaluar los proyectos de desarrollo, así como la propia labor de las entidades de la educación superior en los territorios en pro de la gestión del desarrollo.

\section{Conclusiones}

Las políticas universitarias de ciencia, tecnología e innovación descansan en fundamentos epistemológicos y axiológicos muy diversos. Lo que se considera conocimiento relevante y las políticas para generarlo dependen de una constelación de valores e intereses que desbordan los límites de las universidades. Así, por ejemplo, el acento en el papel económico del conocimiento ha conducido al énfasis en la llamada "tercera misión". Más recientemente han comenzado a influir políticas que sin ignorar la importancia del conocimiento universitario para fines de competitividad y con ello la importancia de las ar- 
ticulaciones universidad-empresa, están incorporando a sus agendas de CTI y formación, objetivos directamente vinculados a la inclusión, la cohesión y la integración social. En esa proyección se espera que las universidades contribuyan a la construcción de sistemas de innovación que generen no solo riqueza sino también inclusión social.

Hemos sugerido en este documento que la trayectoria de la educación superior cubana puede ser vista como un esfuerzo por construir capacidades en CTI y alinearlas con las necesidades del desarrollo a través de la solución de problemas económicos y sociales y la creación de capacidades.

Los cambios ocurridos en las políticas institucionales los hemos resumido en un conjunto de "giros. A través del conjunto de los "giros": "giro a la investigación", "giro a políticas orientadas a la investigación aplicada", "giro a la innovación", "giro al posgrado" y el "giro territorial", las universidades cubanas han intentado en diversos momentos y contextos actuar como "universidades desarrolladoras".

La política orientada a fortalecer el nexo educación superior-desarrollo local, territorial es la demanda que moviliza a los actores involucrados en Programa GUCID. La recuperación de la economía local, el mejoramiento de la administración y el gobierno, la solución de problemas sociales y ambientales con consecuencias en términos de inclusión, cohesión e integración social, son objetivos inscritos en la agenda de GUCID,

Alientan a GUCID las proyecciones que ha elaborado el país recientemente y que suponen un mayor protagonismo de los actores locales con la correspondiente descentralización de funciones, autoridad y recursos; la necesidad para ello de fortalecer la capacidad institucional y de conocimientos; la urgencia de fortalecer la economía local, así como la necesidad de de crear capacidades en los actores locales para realizar gestión estratégica a través de la formulación, implementación, evaluación, de políticas, programas y proyectos.

Los obstáculos son muchos y provienen en gran medida del hecho de que esas decisiones son recientes y aún es incipiente su plasmación en regulaciones y prácticas.

Desde hace dos años el Ministerio de Educación Superior incluyó en su planeación estratégica el objetivo de fomentar el desarrollo local. Se trata de una visión relativamente novedosa que incluye nuevas políticas y nuevos instrumentos, indicadores entre ellos.

La novedad, y a la vez el desafío, consisten en que las instituciones de educación superior están aprendiendo a operar en la escala local y están ajustando sus capacidades de conocimiento e innovación a las exigencias que de ello se derivan. 


\section{Notas Bibliográficas}

(1) A pesar de la importancia que aquí le atribuimos a las actividades de CTI en las universidades, son apreciables los obstáculos. Ver "Universidades como centros de investigación y creación de conocimiento: ¿una especie en extinción?” (París, UNESCO, 29 de noviembre-1 de diciembre 2006) http://www.unesco.org/education/researchforum

(2) En ella se adoptó un plan de acción extraído de la Declaración Final que subraya el papel de la educación superior en el propósito de: "Construir una agenda regional de Ciencia, Tecnología e Innovación para la superación de brechas y para el desarrollo sostenible de ALC". En uno de los documentos de la conferencia VESSURI, H. et ál.(2008) defiende la idea de una "ciencia de la sosteniblidad" "Un sistema científico orientado a favorecer el desarrollo sostenible desde la perspectiva de un sistema socio-ecológico, caracterizado por su visión holística, interdisciplinaria, vinculado a las decisiones políticas que permitan manejar el riesgo, articulador de saberes diversos y que permita atender los problemas sociales, económicos y ambientales, reduciendo el hambre, la pobreza y la inequidad, a la vez que mantiene la biodiversidad y los sistemas de soporte de la vida en el planeta".

(3) El avance de las universidades latinoamericanas dentro de este modelo puede encontrarse en Schwartzman, S. (ed.) (2008)

(4) Goransson, B and C. Brundenius, C. (eds) (2011): Universities in transition. The changing role and challenges for academic institutions, IDRC, Springer, Ottawa, $366 \mathrm{pp}$. y Goransson, B and C. M. Palsson (eds) (2011) Biotechnology and innovation systems. The role of public policy, IDRC, Ottawa, $399 \mathrm{pp}$.

(5) La Reforma es parte de un proceso más amplio orientado a crear una base científica nacional que tuvo gran importancia en los 60 s y 70 s. Los protagonistas principales del proceso revolucionario concibieron que la Reforma Agraria, la industrialización del país y muchas otras transformaciones en curso necesitaban un tipo de universidad que fuera capaz de promover la ciencia y la tecnología y las articulara al cambio social que tenía lugar. Esa percepción sobre el papel del conocimiento en el desarrollo se expandía por el mundo en la década de los 60 s y sin duda influyó en la joven vanguardia política. Los profesores que permanecieron en las universidades y los estudiantes que comenzaron a incorporarse a ella, con frecuencia procedentes de sectores populares, participaron activamente en el proceso de transformación académica. En la década de los 60 s se multiplicaron los estudios en ciencias e ingenierías, se incrementó la cooperación internacional y se crearon grupos de investigación. La Universidad se vinculó activamente a los planes económicos y sociales.

(6) Cuba ingresó al CAME en 1972 El ingreso en 1972 al Consejo de Ayuda Mutua Económica (CAME) formado por los países del bloque socialista tuvo grandes consecuencias. Este esquema de cooperación perseguía equilibrar los niveles de desarrollo de los países miembros. Cuba recibió las tecnologías que su desarrollo demandaba. Pero mantuvo un patrón de especialización en la producción de bienes, caracterizados como de baja intensidad tecnológica. Este hecho y la intensa transferencia tecnológica desde esos países, sobre todo a partir de 1972, redujo la ambición tecnológica que se había manifestado en la década anterior (Montalvo, 1998).

(7) El Programa Ramal "Gestión Universitaria del conocimiento y la innovación para el desarrollo" (GUCID) es una iniciativa del Ministerio de Educación Superior (MES) que cuenta con el respaldo académico y organizativo de la Cátedra Ciencia, tecnología, sociedad e innovación (CTS+I) de la Universidad de La Habana. GUCID nació en 2006.

(8) Según el autor: "el principal atributo del modo emergente es la generación de conocimiento en el contexto de su aplicación e implicaciones, de lo que resultan sus otras 
características: esfuerzo transdisciplinario, inclusión de la participación de la diversidad de actores y organizaciones del contexto, conocimiento socialmente apropiado en el proceso de su generación, alto contenido ético derivado de su reflexividad y compromiso sociales y control social ampliado sobre la calidad del conocimiento generado y sobre la validez de sus impactos." (Souza, Cheaz y Calderón, 2001).

\section{Referencias Bibliografícas}

- $\quad$ Albornoz, M; López Cerezo, J.A (eds.) (2010). Ciencia, Tecnología y Universidad en Iberoamérica. Buenos Aires: OEl/Eudeba.

- $\quad$ Etzkowitz, H y Leydesdorff, L. (1997). University and the Global Knowledge Economy. A Triple Helix of University-Industry-Government Relations. London: Pinter Publishers.

- Gazzola, A y Didriksson, A. (eds.) (2008). Tendencias de la Educación Superior en América Latina y el Caribe. Caracas: IESALC.

- $\quad$ Gibbons, M. et ál. (1997). La nueva producción del conocimiento. Barcelona: Ediciones Pomares-Corredor.

- Lage, A. (2006). La economía del conocimiento y el socialismo (II): reflexiones a partir del proyecto de desarrollo territorial de Yagüajay. En Núñez, J. (Coordinador) Los marcos conceptuales del programa ramal: en busca de consensos. Documentos a debate. Panel 2 realizado en I Seminario Nacional del Programa Ramal Gestión universitaria del conocimiento y la innovación- GUCID, La Habana, Cuba.

- $\quad$ Lynn Meek, V; U. Teichler, U y Kearney M-L. (eds.) (2009). Higher Education, Research and Innovation: Changing Dynamics. Kassel: UNESCO/INCHER.

- Lundvall, B-A. (2000). Los Sistemas Nacionales de Innovación: relaciones y aprendizaje. En Los Sistemas de Ciencia e Innovación Tecnológica. La Habana: Ediciones del Ministerio de la Industria Básica.

- Montalvo, L.F (1998). La política científica y tecnológica en Cuba: evolución y elementos para su perfeccionamiento. Tesis de Doctor en Política Científica y Tecnológica, Campinas, Sao Paulo, Brasil, 1998.

- Núñez, J. (2010). Conocimiento académico y sociedad. Ensayos sobre política universitaria y posgrado. La Habana: Editorial Universidad de La Habana.

- Núñez, J., Benítez, F., Hernández, D., y Fernández, A. (2008). Universal higher education and sustainable social development: the Cuban model. The International Journal of Cuban Studies, 1(1), June.

- Núñez, J.; Castro, F. (2005). Universidad, innovación y sociedad: experiencias de la Universidad de la Habana. Revista de Ciencias de la Administración, 13 (7), 9-30.

- Núñez, J., Montalvo, L.F; Pérez, I (2007). Universidad y desarrollo social basado en el conocimiento: nuevas estrategias desde lo local. En Gallina, A., Núñez, J., Capecchi, V. Montalvo, L. (Comp.) Innovaciones creativas y desarrollo humano. (pp. 165-184). Florianópolis, Brasil.

- OEl (2012) Ciencia, tecnología e innovación para el desarrollo y la cohesión social. Programa iberoamericano en la década de los bicentenarios. Documento de trabajo. http://www.oei.es

- Santelices, B. (editor-coordinador) (2010). El rol de las universidades en el desarrollo científico y tecnológico. Educación superior en Iberoamérica Informe 2010, Centro Interuniversitario de Desarrollo (CINDA) - Chile: Universia.

- Schwartzman, S. (ed.) (2008). Universidad y desarrollo en Latinoamérica. Experiencias exitosas de centros de investigación. Caracas: IESALC. 
- Souza, J., Cheaz, J; Calderón, J. (2001). La cuestión institucional: de la vulnerabilidad a la sostenibilidad institucional en el contexto del cambio de época. Serie Innovación para la Sostenibilidad Institucional. San José, Costa Rica: Proyecto ISNAR "Nuevo paradigma".

- Sousa Santos, B. (2008). La Universidad en el siglo xxı. Caracas: Centro Internacional Miranda.

- Sutz, J. (2010). Ciencia, Tecnología, Innovación e Inclusión Social: una agenda urgente para universidades y políticas. Psicología, Conocimiento y Sociedad Número 01. Revista de la Facultad de Psicología Investigación e inclusión social.

- Vessuri, H. (2008). El futuro nos alcanza: mutaciones previsibles de la ciencia y la tecnología. En Gazzola, A y Didriksson, A (eds.). Tendencias de la Educación Superior en América Latina y el Caribe. Caracas: IESALC. pp.55-86. 